Supplement of Biogeosciences, 18, 2727-2754, 2021 https://doi.org/10.5194/bg-18-2727-2021-supplement (C) Author(s) 2021. CC BY 4.0 License.

(c) (1)

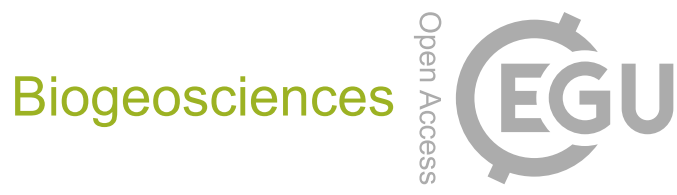

Supplement of

\title{
Optimal model complexity for terrestrial carbon cycle prediction
}

Caroline A. Famiglietti et al.

Correspondence to: Caroline A. Famiglietti (cfamigli@stanford.edu)

The copyright of individual parts of the supplement might differ from the article licence. 
(a) Model: S2

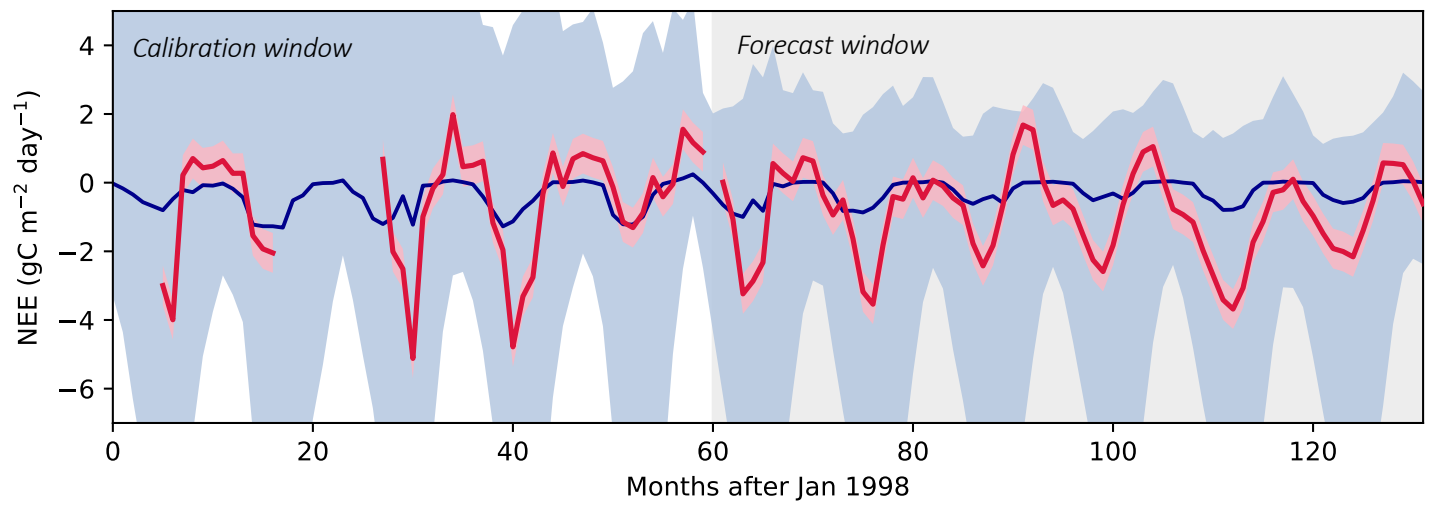

(b) Model: C2 Assimilated data: None Error scalar: N/A EDCs: Off

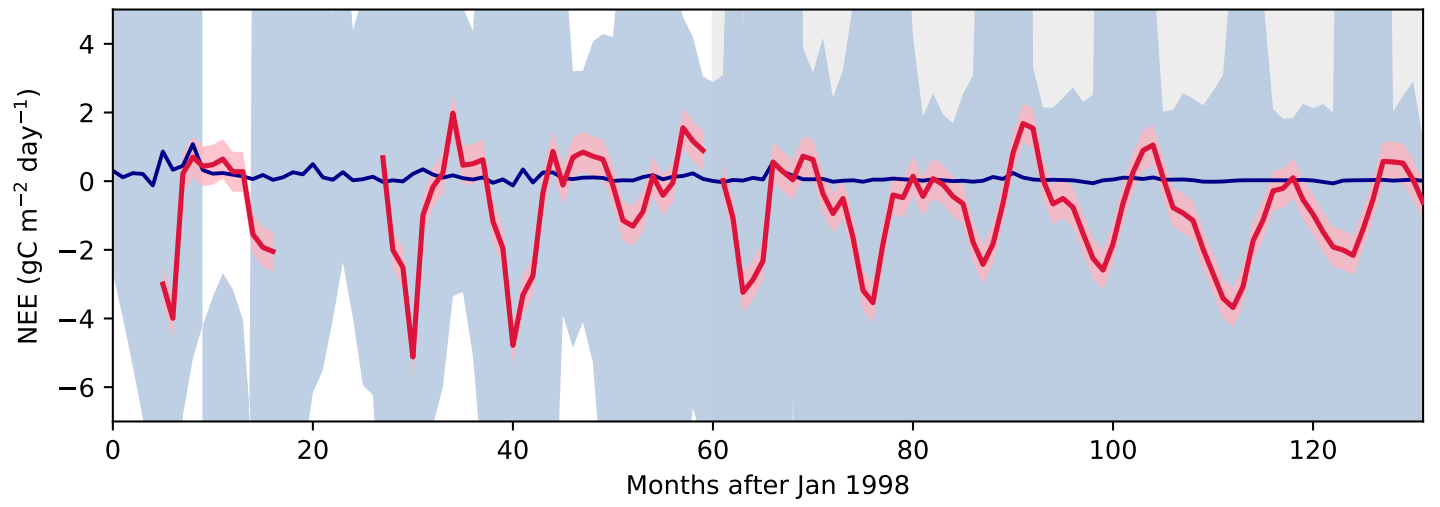

(c) Model: G4 Assimilated data: None Error scalar: N/A EDCs: Off

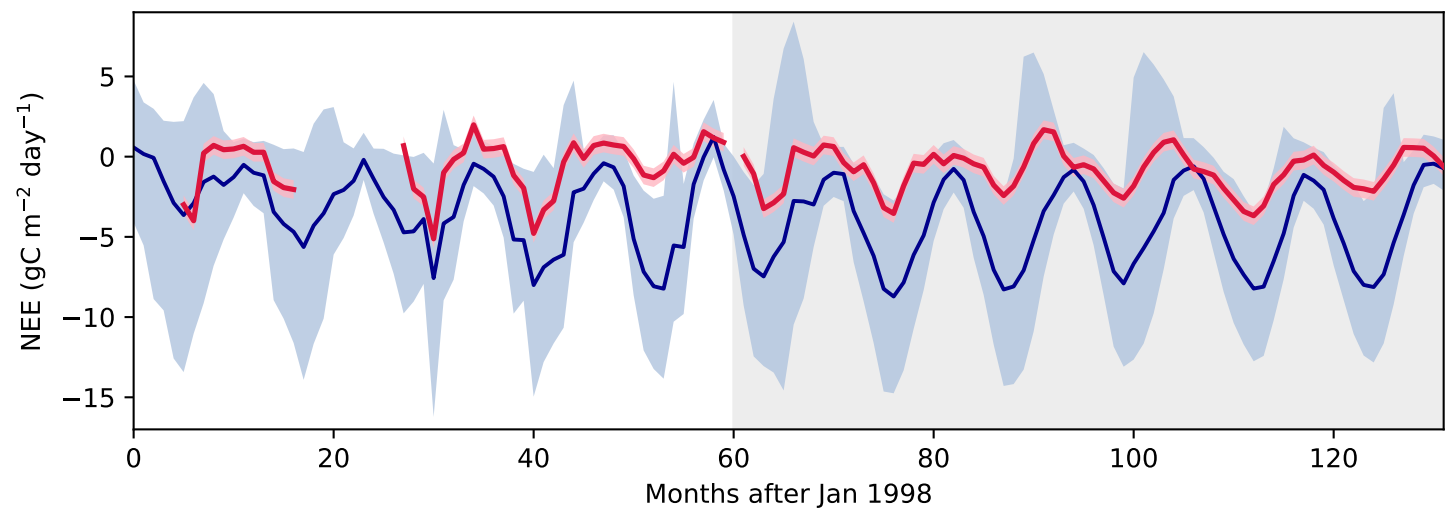

\section{- Predicted Ensemble spread - Observed Observational uncertainty}

Fig. S1: Example model runs parameterized strictly using prior distributions at the FR-LBr site. For comparison, panels 20 correspond directly to the models shown in Fig. 3. The calibration window-the first 5 years of the record-is shown in white and the forecast window is shaded gray. The ensemble spread (blue shading) encapsulates the 5th-95th percentile of runs. 


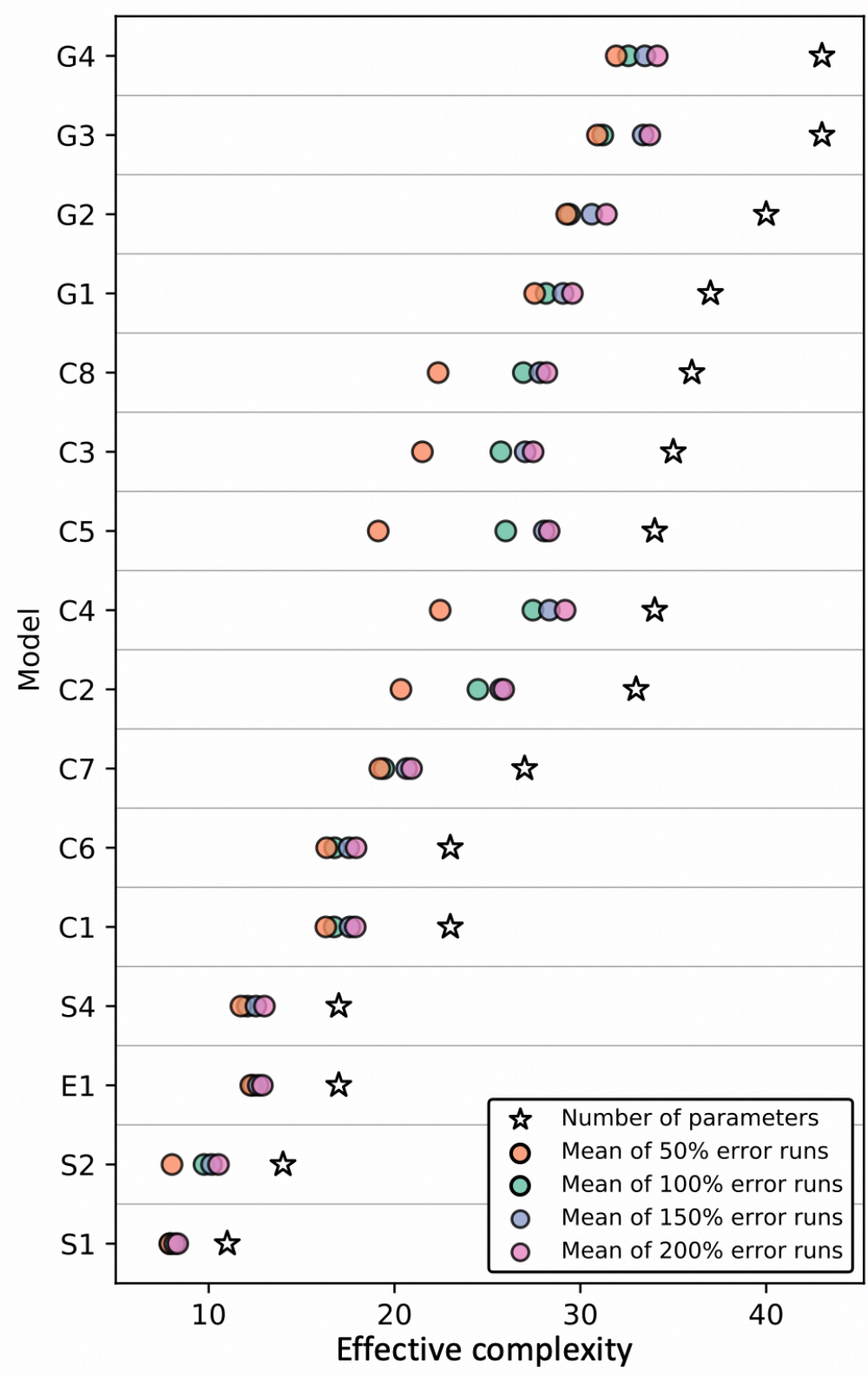

Fig. S2: Effect of observational error scalar on effective complexity. Models are ordered from fewest (S1) to 25 greatest (G4) number of parameters. 


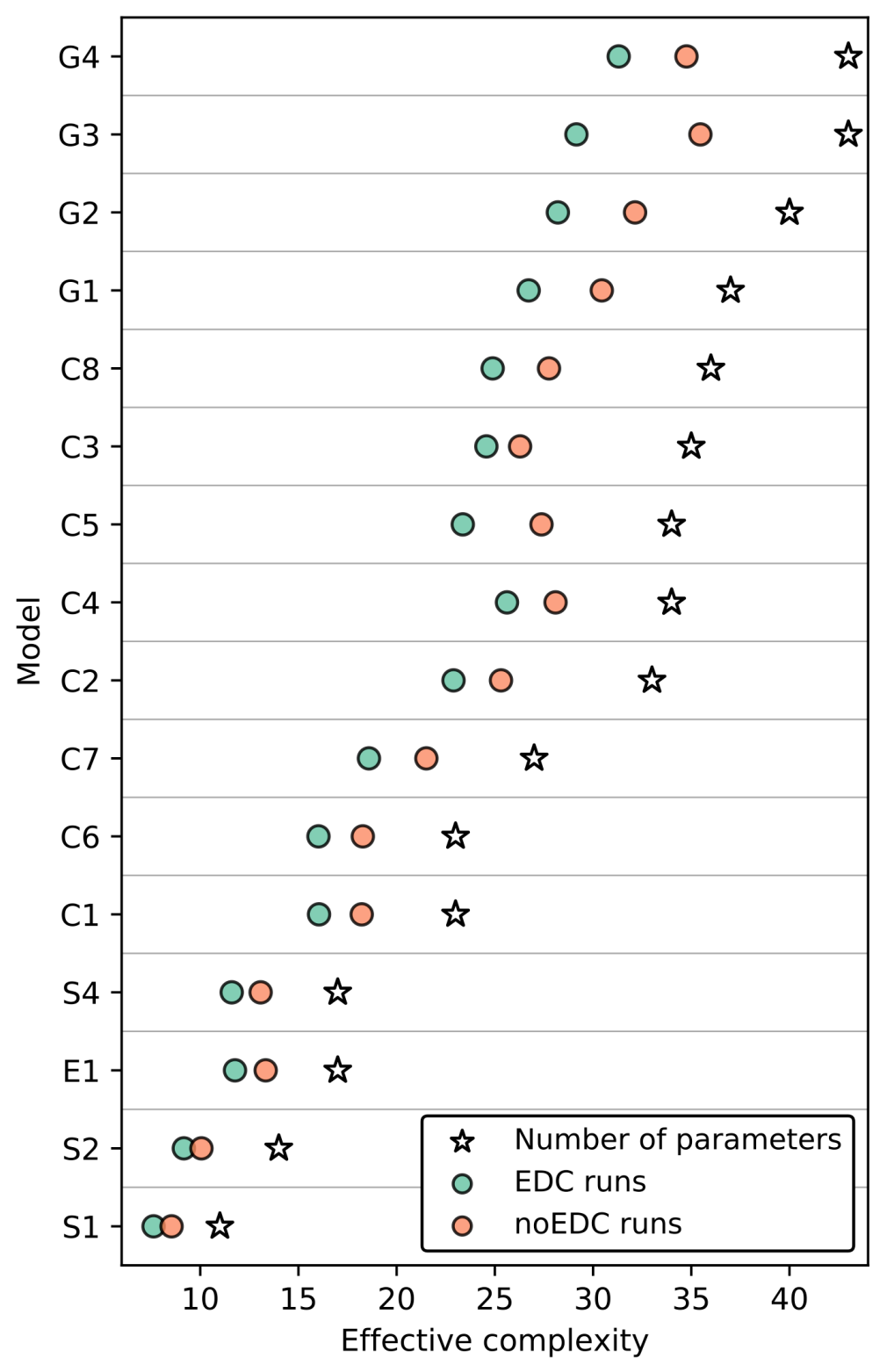

Fig. S3: Effects of EDCs on effective complexity. Models are ordered from fewest (S1) to greatest (G4) number of parameters. 
(a) All runs

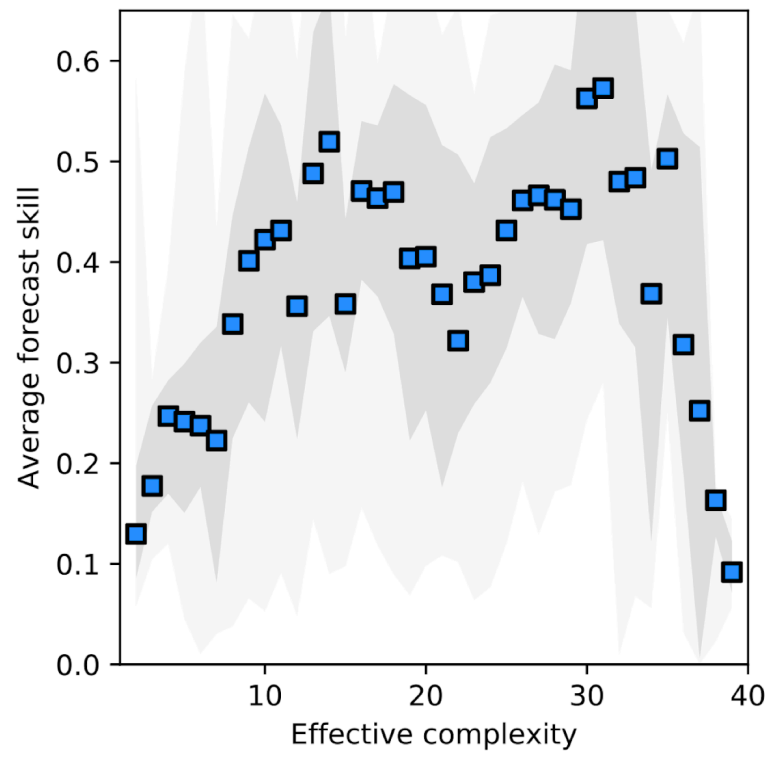

(b) All runs with assimilated data

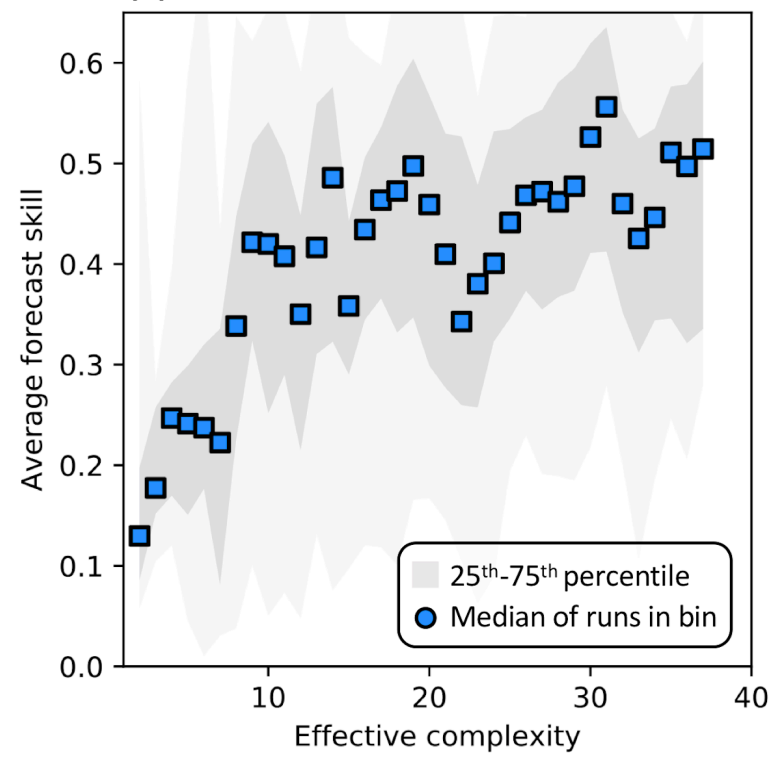

30 Fig. S4: Complexity-skill relationship for LAI predictions. (a) All model runs included in the experiment; (b) all model runs for which data was assimilated. Dark gray shading spans the $25^{\text {th }}$ to $75^{\text {th }}$ percentile of runs; light gray shading spans $5^{\text {th }}$ to $95^{\text {th }}$ percentile; blue points are medians of complexity bins. Average forecast skill is computed using the histogram intersection metric. 
(a) All runs in the experiment

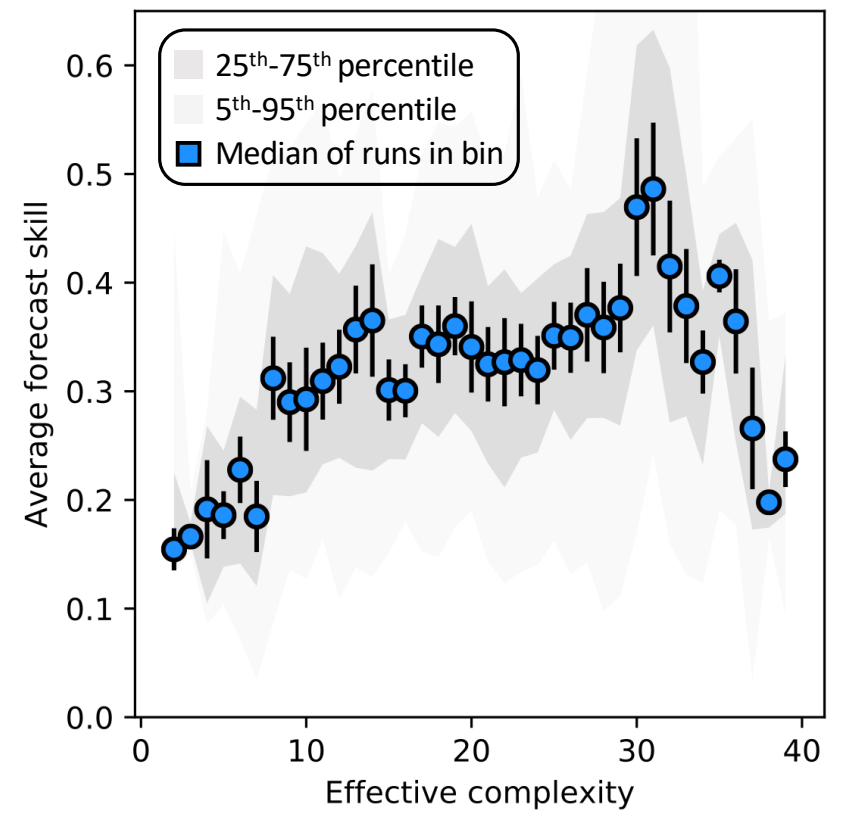

(b) All runs with assimilated data

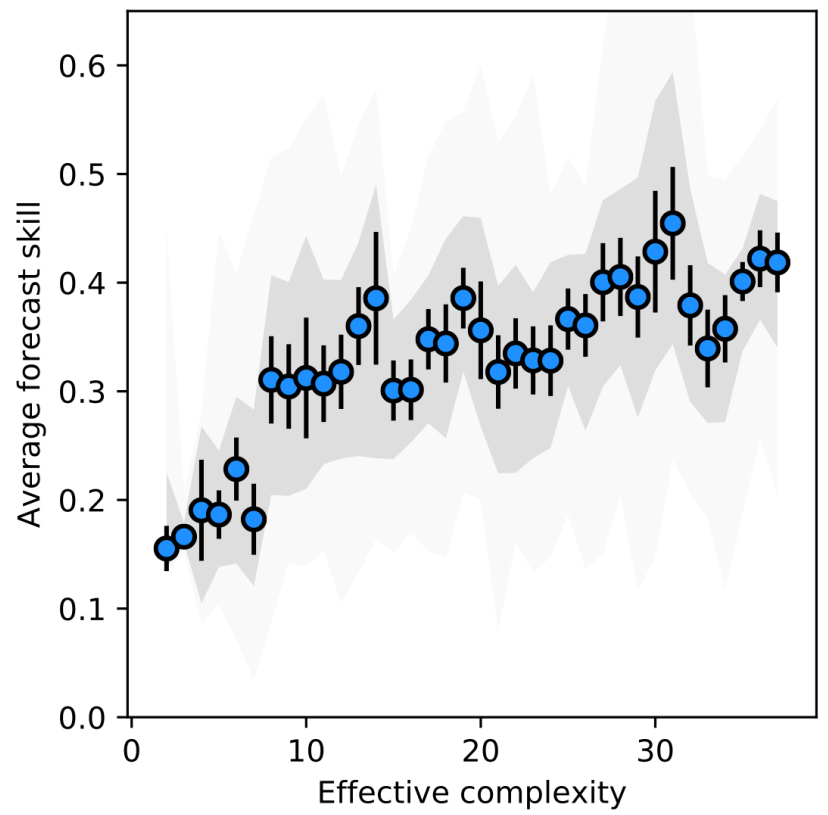

Fig. S5: Complexity-skill relationship for NEE predictions, where a bootstrapping procedure has been performed to equalize number of runs within each complexity bin. Error bars represent one standard deviation of skill across the different bootstrap combinations. 
(a) $50 \%$ error

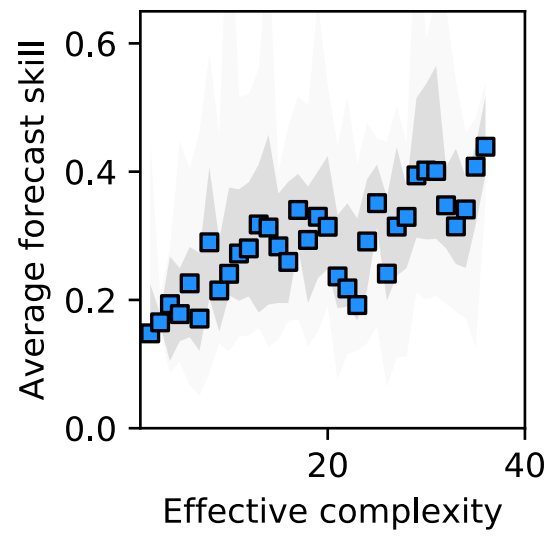

(c) $150 \%$ error

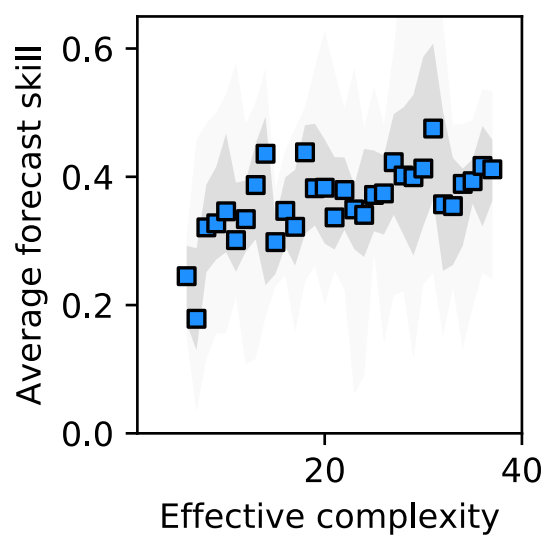

(b) $100 \%$ error

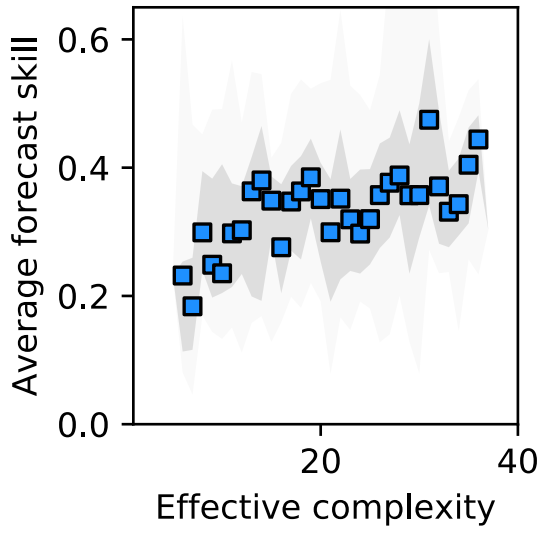

(d) $200 \%$ error

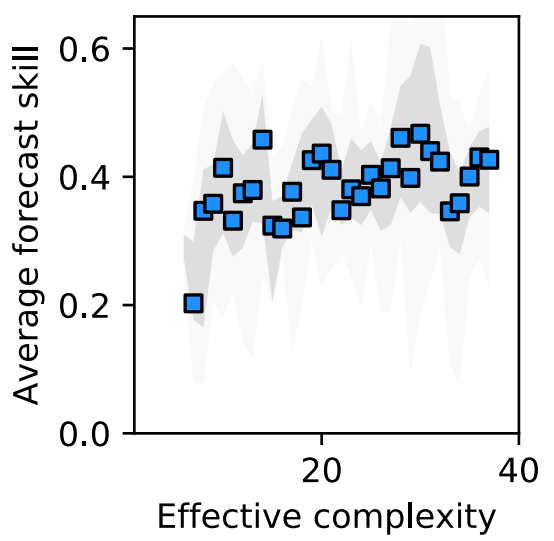

$25^{\text {th }}-75^{\text {th }}$ percentile

$5^{\text {th }}-95^{\text {th }}$ percentile

$\square$ Median of runs in bin

Fig. S6: Complexity-skill relationship for NEE predictions, split by observational error scalar (title of each subplot). Dark gray shading spans the $25^{\text {th }}$ to $75^{\text {th }}$ percentile of runs; light gray shading spans $5^{\text {th }}$ to $95^{\text {th }}$ percentile; 45 blue points are medians of complexity bins. Average forecast skill is computed using the histogram intersection metric. 
(a) NEE, LAl, biomass

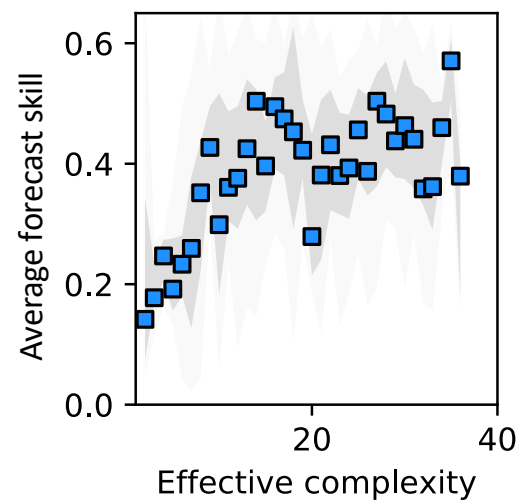

(d) LAI, biomass

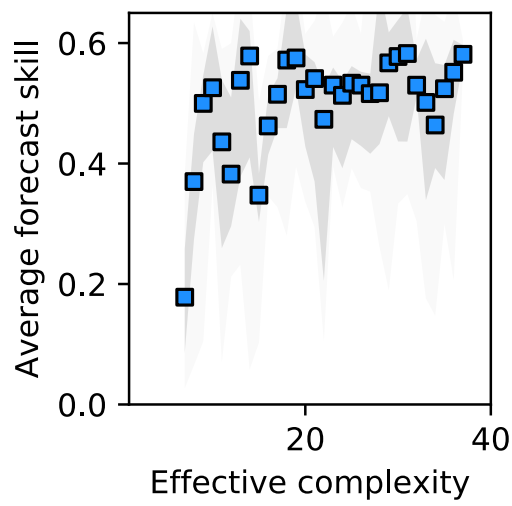

(b) NEE, LAI

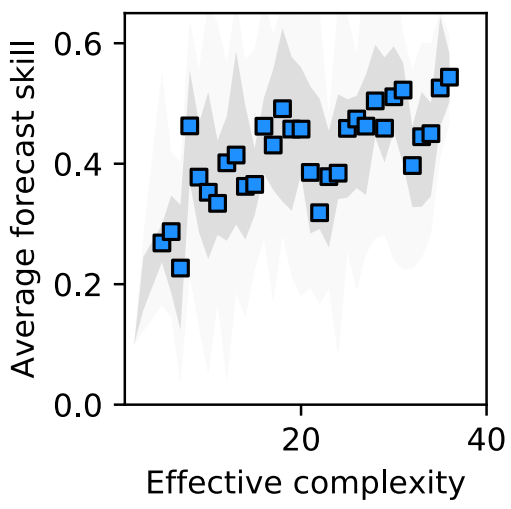

(e) LAI

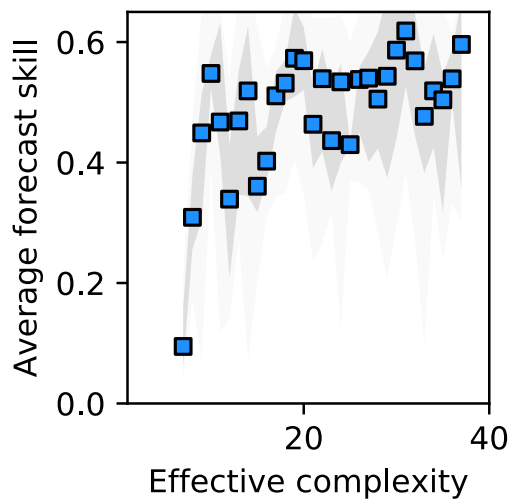

(c) NEE

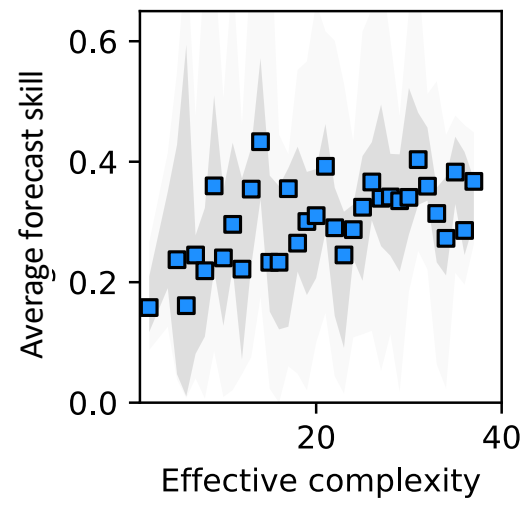

(f) None

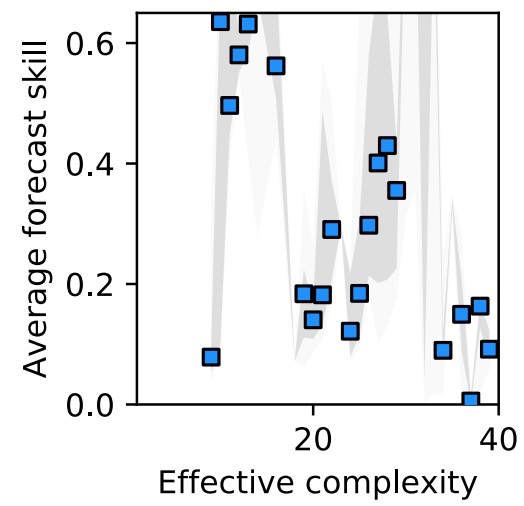

$25^{\text {th }}-75^{\text {th }}$ percentile

$5^{\text {th }}-95^{\text {th }}$ percentile

Median of runs in bin

Fig. S7: Complexity-skill relationship for LAI predictions, split by assimilated data subset (title of each subplot). Dark gray shading spans the $25^{\text {th }}$ to $75^{\text {th }}$ percentile of runs; light gray shading spans $5^{\text {th }}$ to $95^{\text {th }}$ percentile; blue points are medians of complexity bins. Average forecast skill is computed using the histogram intersection metric. Ordering of subplots reflects strongest (a) to weakest (f) data constraint. 